\title{
Velocity profiles of fluid flow close to a hydrophobic surface
}

\author{
Simona Fialová ${ }^{1}$, František Pochylý ${ }^{1}$, Michal Kotek $^{2}$, Darina Jašíková \\ ${ }^{1}$ Faculty of mechanical engineering, Brno University of Technology, Technicka 2896/0, Kralovo Pole, 61669, Brno, Czech \\ Republic \\ ${ }^{2}$ Department of Physical Measurement, Technical University of Liberec, The Institute for Nanomaterials, Advanced \\ Technology and Innovation, Studentska 1402/2 Liberec 1461 17, Czech Republic
}

\begin{abstract}
The results of research on viscous liquid flow upon a superhydrophobic surface are presented in the paper. In the introduction, the degrees of surface hydrophobicity in correlation with an adhesion coefficient are defined. The usage of the adhesion coefficient for the definition of a new boundary condition is employed for expressing the slip of the liquid over the superhydrophobic surface. The slip of the liquid was identified on a special experimental device. The essence of the device consists of a tunnel of rectangular cross section whose one wall is treated with a superhydrophobic layer. The other walls are made of transparent organic glass whose surface is hydrophilic. Velocity profiles are measured by PIV. The methodology is drawn so that it allows the speed determination at the closest point to the wall. The measurements were performed for different Reynolds numbers for both laminar and turbulent flow. Based on the measured velocity profiles, marginal terms of use have been verified, expressing slippage of the liquid on the wall. New forms of velocity profiles considering superhydrophobic surfaces are shown within the work.
\end{abstract}

\section{Introduction}

It is nowadays possible to generalize the solution of hydrodynamic problems and assume hydrophobic character of the surface in the solid/liquid interaction studied by [5], [7], [8]. This characteristic of the surface to repel liquid was assumed by Navier [9], 200 years ago. For liquid on hydrophobic surface, he predicted the shear stress to be proportional to the slipping velocity.

The findings from experimental research on superhydrophobic surfaces is presented in the paper. Experiments describing the flow behavior over the hydrophobic and superhydrophobic surfaces were presented by numerous authors. For example [5], studies the wettability effect on the flow stabilty and the transient process. The slip boundary condition is written that a velocity is proportional to the velocity gradient and the slip lenght.

Calculations on the wall were made by linearized Navier-Stokes equations for 2D flow (OrrSommerfeld equation). Their conclusion states that the transition to turbulent flow is slowed down in the streamline direction and quickend perpandiculary on it. Friction losses were stuied within the work [6], where the PIV (Particle Image Velocimetry) method was used and studied the water flow over superhydrophobic surface in the channel of small crossection. Based on our experiment, a contact angle of water droplets on a flat surface [1], [2], [10] is determined. A methodology for the determination of an adhesion coefficient is outlined [1], [2], [10]. For such case applies the boundary condition $\boldsymbol{\sigma}_{A}=(\boldsymbol{\sigma} \times \boldsymbol{n}) \times \boldsymbol{n}=-k \boldsymbol{v}$ with the rationale and the results of velocity profiles measurements using PIV method being introduced. Using PIV methods, the curves of the velocity profiles in the channel of large rectangular cross section for the various types of superhydrophobic surfaces are measured.

\section{Experimental setup - Contact angles}

The contact angles and adhesion coefficient on superhydrophobic (SH) surfaces was measured using optical shadowgraphy methods. The steel sample was situated between the tabular LED panel and the digital camera. The LED light pointed over the sample straight to the camera. The droplet on 
the SH surface was represented by a dark shadow object on the white background, see Fig. 2.1.

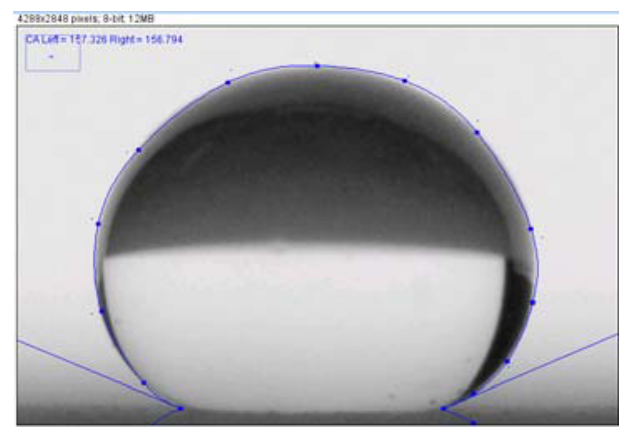

Fig.2.1 Image of a single droplet on SH surface with determined contact angles CA (left and right)

Because of the high background-object contrast and significant object border identification, this method is suitable for object geometry analysis and curve measurement.

The measurement of the contact angle was a static process applied on one image of a single droplet of $1 \mu \mathrm{l}$ to avoid the gravity effect of the droplet wight on the result. The image records were analyzed by a unique processing algorithm. The contact angles were measured on both sides of each droplet (left and right CA). Ten separated droplets were recorded to ensure a reliable result of the contact angle on the surface under investigation. The contact angle measurements were first made on a dry sample, see Tab. 2.1 and then on a wet sample after measurement of the flow in the water channel, see Tab. 2.2. The dry and wet surfaces provided significantly different results for most samples.

Tab.2.1 Measurements of CA on air before measurements in the channel

\begin{tabular}{|c|c|c|}
\hline sample & Left CA & Right CA \\
\hline 1 & 159,07 & 161,01 \\
\hline 2 & 150,88 & 157,09 \\
\hline 3 & 157,45 & 154,65 \\
\hline 4 & 157,25 & 152,37 \\
\hline 5 & 159,63 & 142,95 \\
\hline 6 & 151,06 & 147,13 \\
\hline 7 & 165,98 & 149,74 \\
\hline 8 & 155,90 & 156,33 \\
\hline 9 & 158,97 & 152,88 \\
\hline 10 & 151,31 & 161,23 \\
\hline average & $\mathbf{1 5 6 , 7 5}$ & $\mathbf{1 5 3 , 5 4}$ \\
\hline \pm & 4,49 & 5,53 \\
\hline
\end{tabular}

Tab.2.2 Measurements of CA after measurements in the channel filled with water

\begin{tabular}{|c|c|c|}
\hline sample & Left CA & Right CA \\
\hline 1 & 125,58 & 123,16 \\
\hline
\end{tabular}

\begin{tabular}{|c|c|c|}
\hline 2 & 125,15 & 134,83 \\
\hline 3 & 123,73 & 119,62 \\
\hline 4 & 133,77 & 128,40 \\
\hline 5 & 145,54 & 140,01 \\
\hline 6 & 141,64 & 131,66 \\
\hline 7 & 148,12 & 129,79 \\
\hline 8 & 124,99 & 115,98 \\
\hline 9 & 125,75 & 132,77 \\
\hline 10 & 121,19 & 125,37 \\
\hline average & $\mathbf{1 3 1 , 5 5}$ & $\mathbf{1 2 8 , 1 6}$ \\
\hline \pm & 9,48 & 6,89 \\
\hline
\end{tabular}

\section{Slip coefficient measurement}

Measurement of the slip coefficient was also provided by the optical shadowgraphy method. The metal sample was fixed to a base with an adjustable angle of inclination. Liquid injector was used to drop a droplet to the sample surface; upper Fig. 3.1. When the surface was inclined enough, the droplet started to move on the sample; bottom Fig. 3.1. The acceleration of the droplet was analyzed from the images recorded on a high speed camera. The next series of images shows the process of image preprocessing and analysis of the droplet movement.

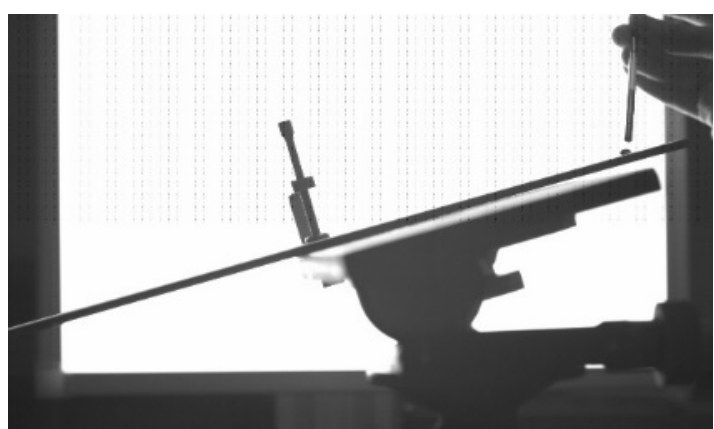

Fig. 3.1 Experiment with the droplet on an inclined plane - original image of the process

The segmentation algorithm was applied on every recorded image . to find the center of each droplet. Images of the droplet movement were recorded with 500 frames per second. The movement of the center of the droplet between every 5 images is calculated.
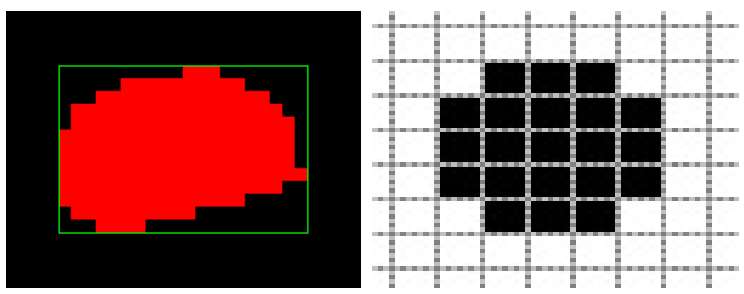

Fig. 3.2 Image preprocessing - obstructive objects elimination 
Using this movement, the instantaneous speed of the droplet is determined. The increase in speed of the droplet gives an information about the acceleration and provides important data to calculate the slip coefficient.
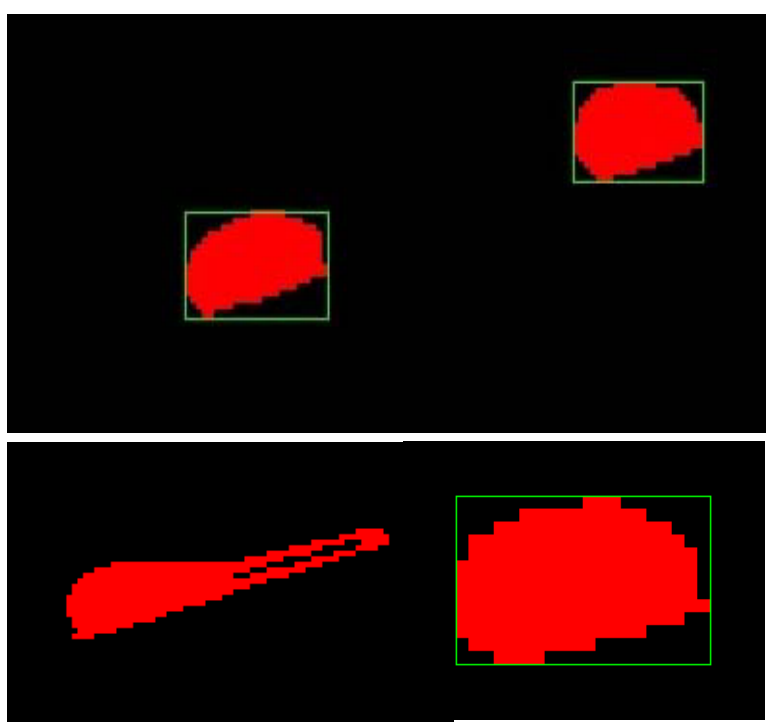

Fig 3.3 Droplet image recognition and tracking process

The adhesion coefficient is finally determined from the mathematical model according to the equation (3.1), based on regression analysis [1], [2]:

$$
\frac{d v_{1}}{d t}=\left(v_{1}-\frac{\left|\sigma_{A}\right|_{\kappa}}{\kappa}\right) e^{-\frac{S \kappa}{m} t}
$$

where $v_{l}$ - velocity of the droplet, $\sigma_{A}$ - shear stress, $\kappa$-adhesion coefficient, $m$ - mass of the droplet, $t-$ time, $S$ - solid/liquid contact surface

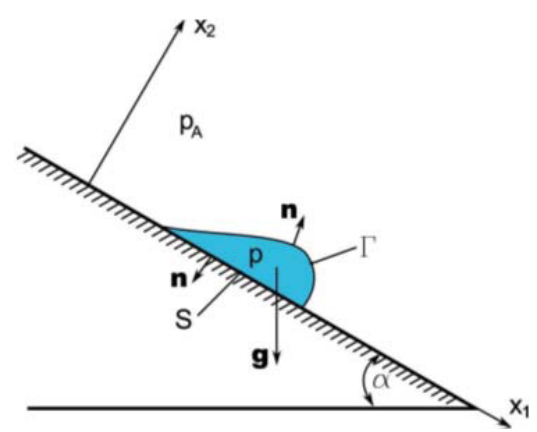

Fig 3.4 Scheme of the droplet on an inclined plane $\mathrm{p}$ - pressure in the droplet, $\mathrm{p}_{\mathrm{A}}$ - atmospheric pressure, $\mathrm{n}$ - nominal vector, $\mathrm{S}$ - solid/liquid contact surface, $\Gamma-$ air/liquid contact surface, $\mathrm{g}$ - gravity acceleration, $\alpha-$ angle of plane inclination

\section{Flow profiles measurement}

Flow profiles above the SH surfaces were measured using Particle Image Velocimetry (PIV) method.
The PIV system was built by Dantec Dynamics, consisting of the high sensitive HighSense NEO camera, NewWave Gemini laser $(100 \mathrm{~mJ}$ on $15 \mathrm{~Hz})$ and Dynamic Studio software for data acquisition and analysis [4]. Images were recorder with the frequency of $4 \mathrm{~Hz}$. The HighSense NEO camera captured the images with the resolution of $2500 \times 2000$ pixels. For PIV correlation analysis, the interrogation areas of $32 \times 32$ pixels were used, ino rder to provide a sufficient spatial resolution of the resulting flow field. The standard PIV analysis was applied on the recorded data. In the first step, the light scattered from walls was masked. After that the double images were correlated using the PIV crosscorrelation method. The vector maps were validated with the Peak and Range validation method. For each flow condition 250 records were captured and statistically analyzed. Amap of mean velocity vectors was used to obtain the profile plot in the selected position.

The setup of the horizontal laser shield was chosen to eliminate the minor temperate influence on the flow in the channel. The scheme of the PIV system setup is shown in Fig. 4.1.

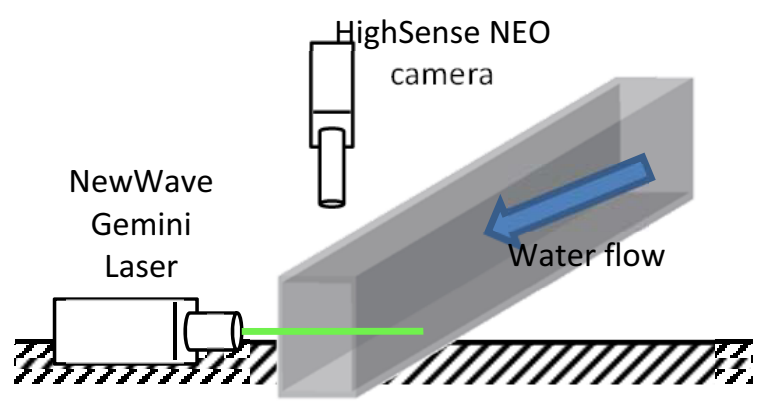

Fig. 4.1 Scheme of the PIV setup for flow profiles measurement

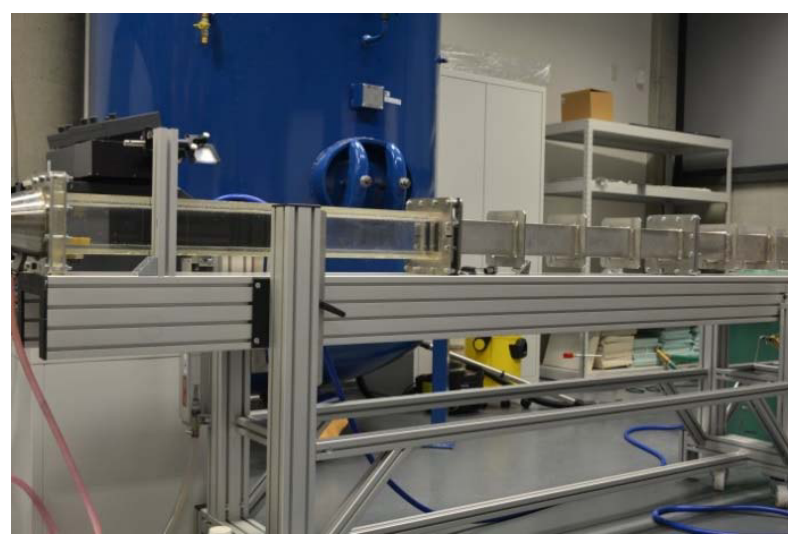

Fig. 4.2 Experimental device for the flow velocity profiles measurements

Emphasis was placed on flow settling in the water channel. The channel has the cross-section dimensions $75 \times 80 \mathrm{~mm}$, so the settling length was established to 4 meters, as is visible from Fig. 4.2. To eliminate pulsations of the flow caused by a water pump, the flow was driven by gravity. A pump 
was used to deliver the water to the top reservoir with the level 2 meters above the experiment and the lower reservoir. The flow was controlled by a valve and measured on an induction flowmeter. The flow profiles were measured in the selected range of Reynolds numbers.

\section{Velocity profiles}

As previously stated, the velocity profiles were measured in the rectangular area, made out of plexiglas (perspex) with a contact angle of approximately $70^{\circ}$, for two tasks. In the first task, all inner walls were hydrophilic (blue line) whereas in the second case, one out of four sides of the channel is provided with a superhydrophobic coating (red line) while the three other walls were wettable. From here the asymmetry of the velocity profile is apparent. A comparison of the velocity profiles was performed at a constant flow rate, always for both variants. The following figures show the velocity profiles in the selected plane, but also the details of velocity profiles near the wall. From the graphs it is apparent that indeed in the vicinity of the wall a slippage of the liquid on the superhydrophobic surface occurs. The velocity of the liquid increases with increasing flow rate. See Figures 5.1 - 5.6; where for all holds that the $\mathrm{x}$-axys is distance (canal width in $\mathrm{mm}$ ) and $\mathrm{y}$-axis is velocity $(\mathrm{m} / \mathrm{s})$.

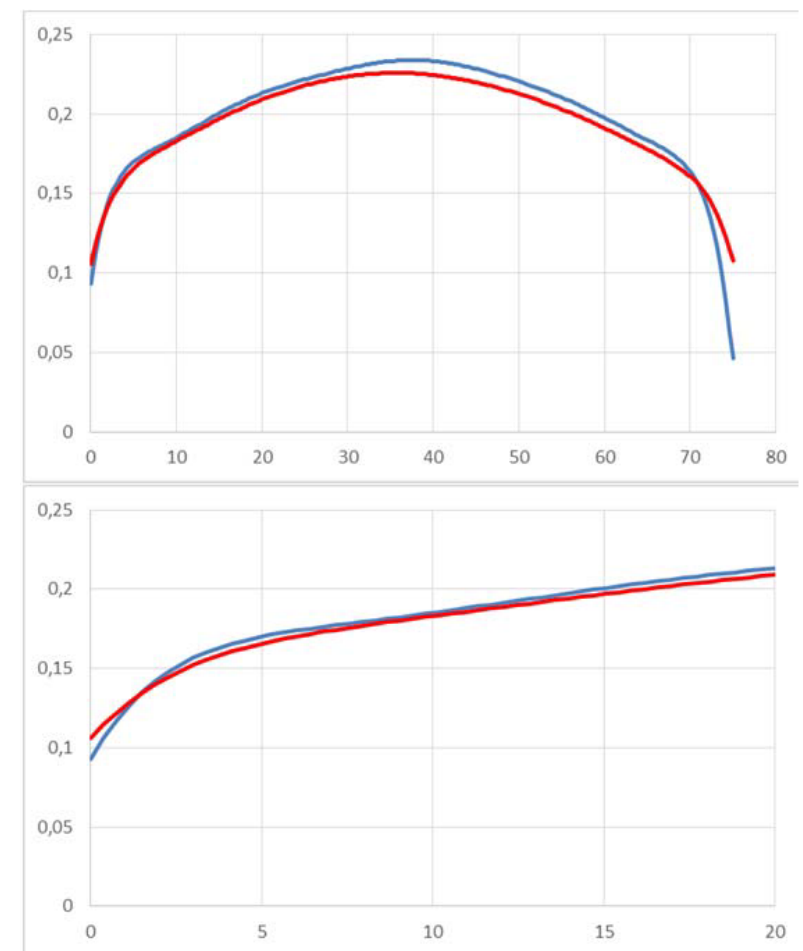

Fig.5.1 Velocity profile and the detail close to the wall for $\mathrm{Re}=13780$ - red superhydrophobic, blue hydrophilic

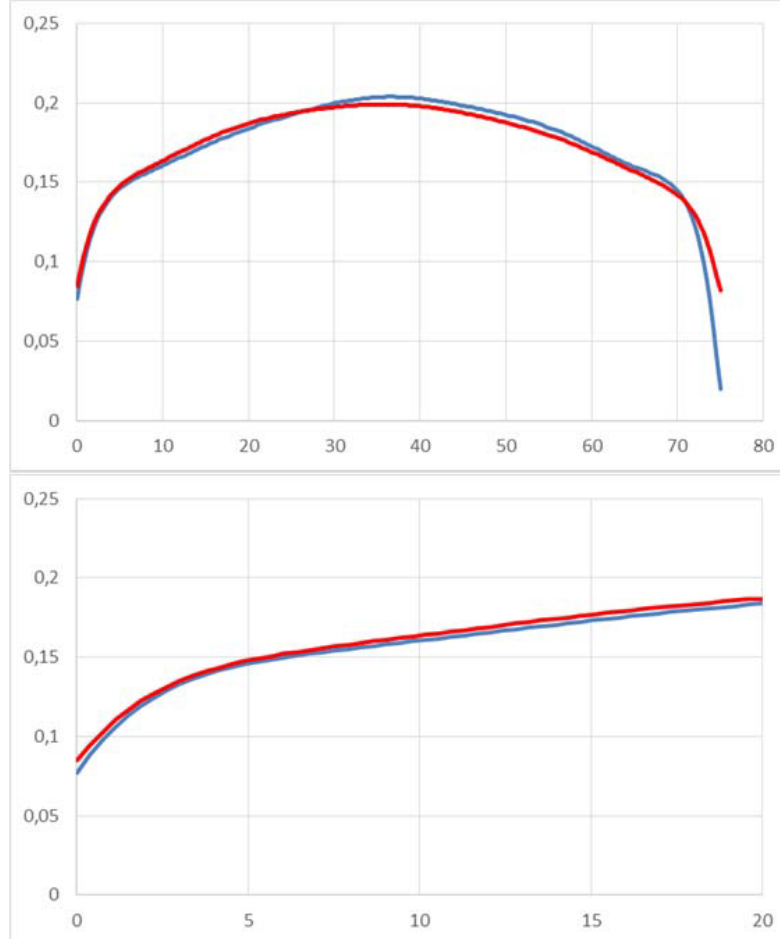

Fig.5.2 Velocity profile and the detail close to the wall for $\mathrm{Re}=12000-$ red superhydrophobic, blue hydrophilic

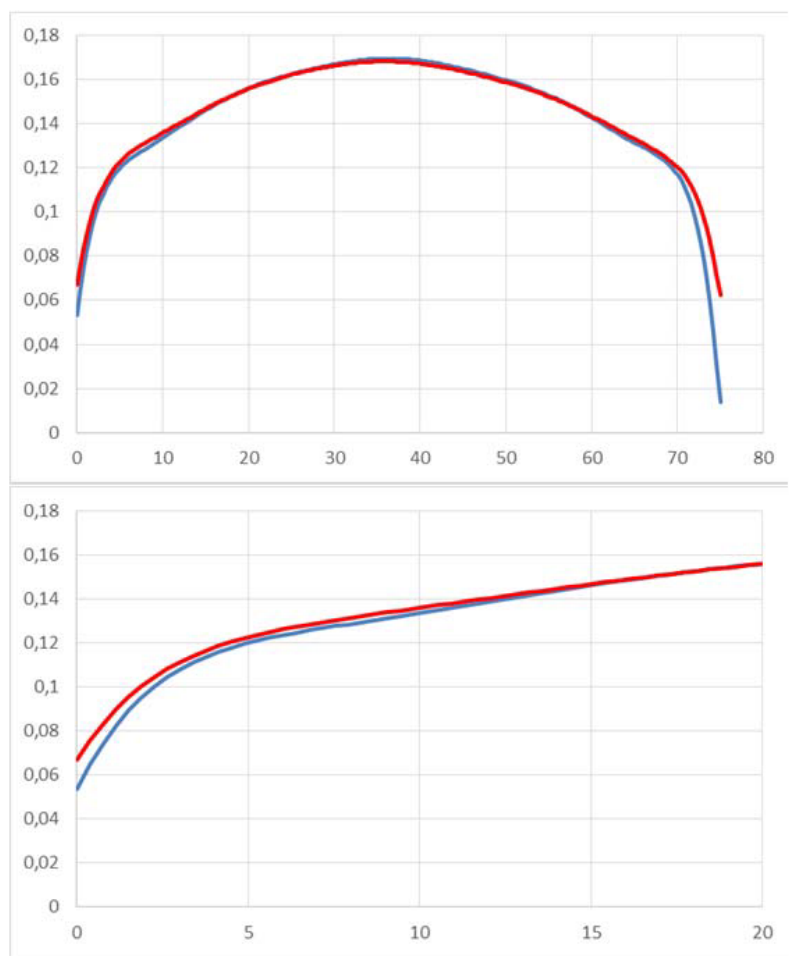

Fig.5.3 Velocity profile and the detail close to the wall for $\mathrm{Re}=10000-$ red superhydrophobic, blue hydrophilic 


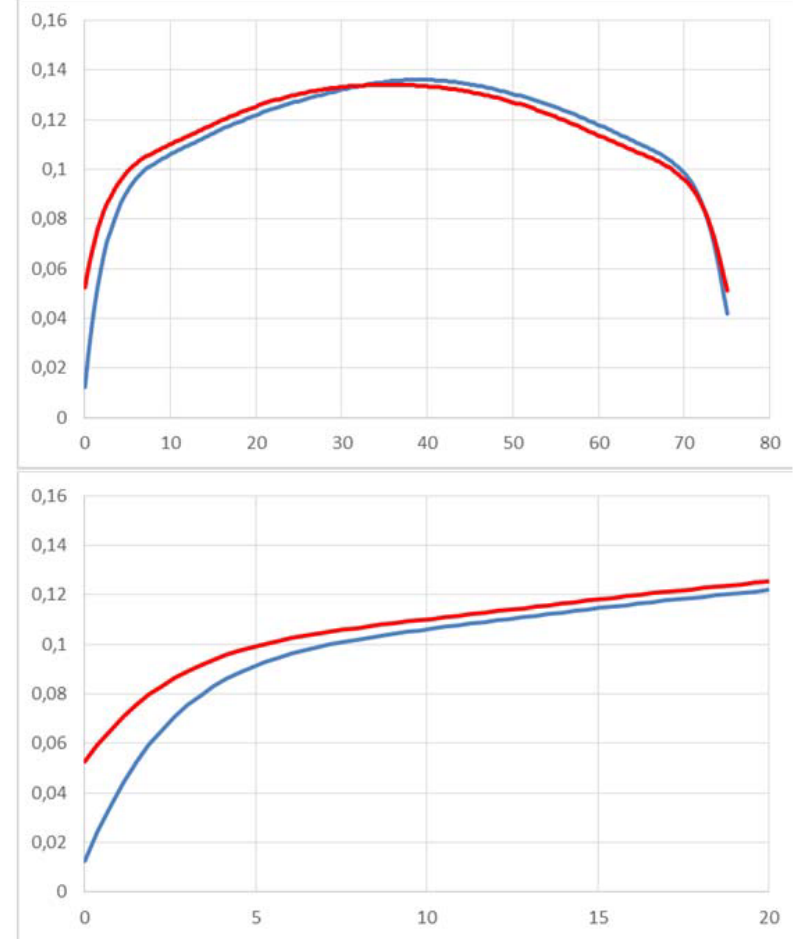

Fig.5.4 Velocity profile and the detail close to the wall for $\operatorname{Re}=8000$ - red superhydrophobic, blue hydrophilic

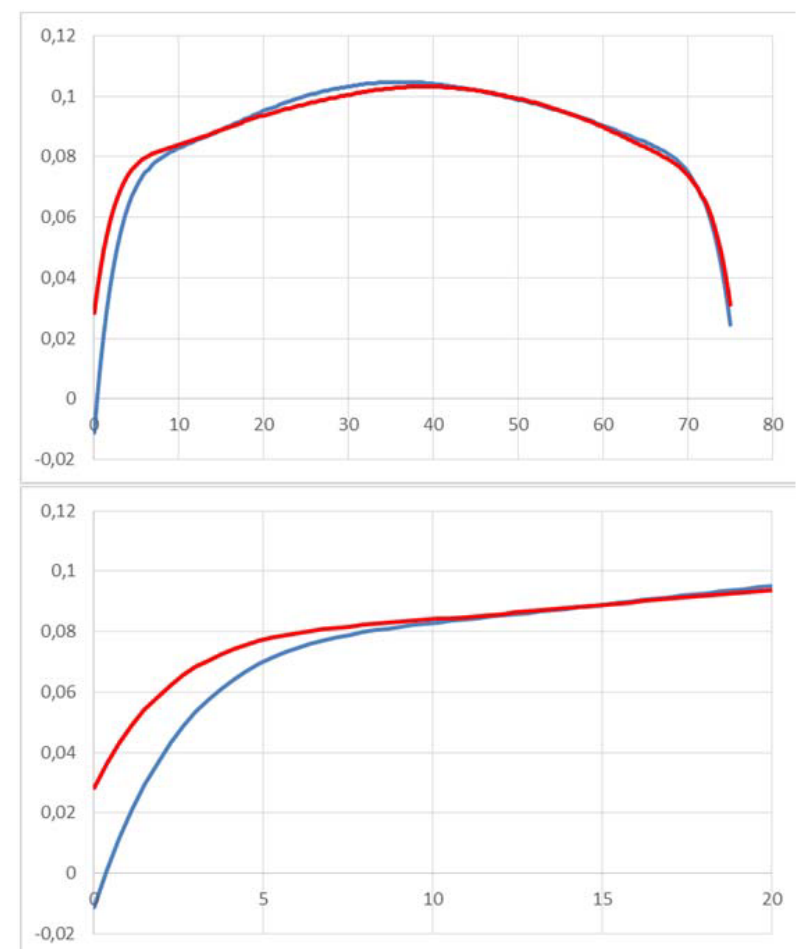

Fig.5.5 Velocity profile and the detail close to the wall for $\operatorname{Re}=6000$ - red superhydrophobic, blue hydrophilic

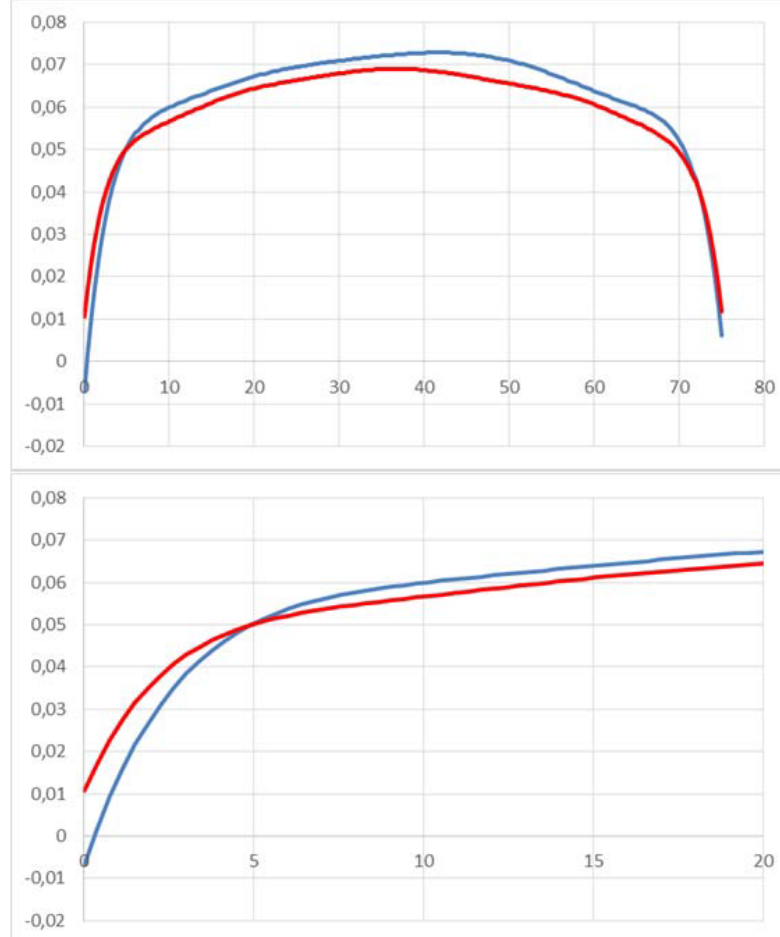

Fig.5.6 Velocity profile and the detail close to the wall for $\operatorname{Re}=4000$ - red superhydrophobic, blue hydrophilic

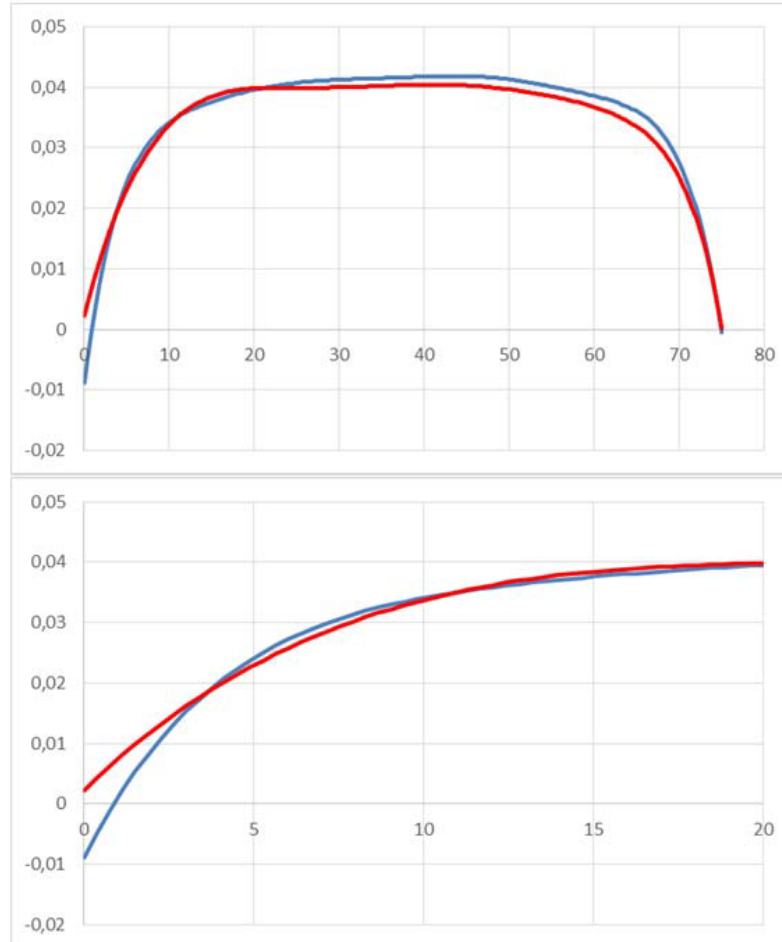

Fig.5.7 Velocity profile and the detail close to the wall for $\operatorname{Re}=2000-$ red superhydrophobic, blue hydrophilic

\section{Conclusion}

The methodology for the measurement of the contact angle of water droplets on a superhydrophobic surface was presented within this 
work. The adhesion coefficient and velocity profiles with the implementation of superhydrophobic surfaces were shown [3]. Measurements demonstrate the slip of the liquid along the superhydrophobic surface with a contact angle of approximately $140^{\circ}$. The contact angle did not change significantly during the experiments. The curves of the velocity profiles are shown in Fig 5.1 - 5.7., where the velocity profile near the wall is highlighted. From the figures the slippage of the liquid is apparent close to the superhydrophobic surface. From this we can obtain the velocity slip value that is needed for the computations of the flow affected by hydrophobic surfaces. The measured curves of the velocity profiles were evaluated based on regression analysis. Fig. 6.1 shows the originally measured velocity profile curves near the wall. Obtained data presented within the graphs were used for the aim of the dissipative energy setting and from the approximations we can obtain the value of the liquid slippage on the wall.

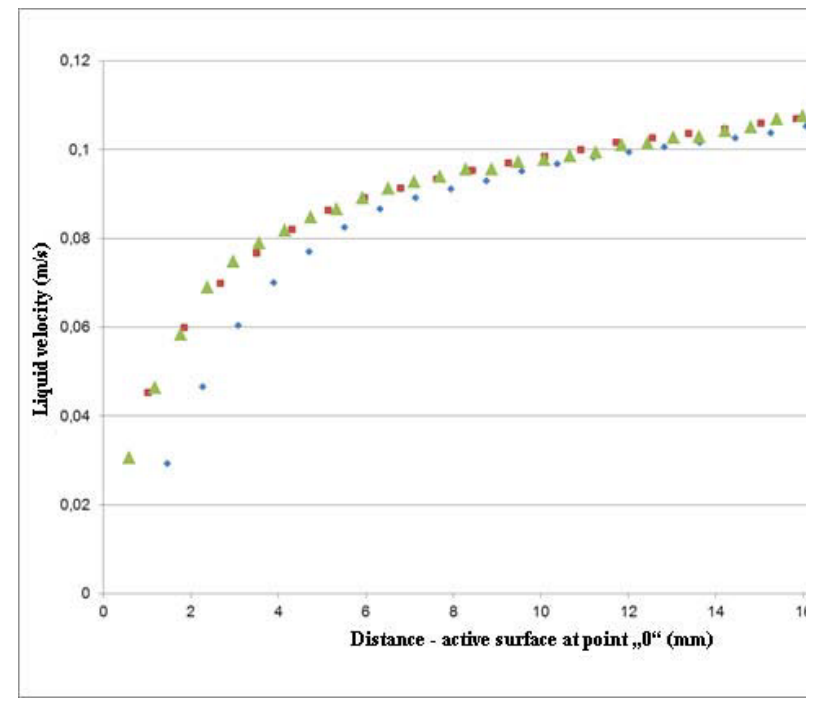

Fig. 6.1 Water velocity profiles close to the superhydrophobic surface (blue dots- plexiglas, CA $\theta=80^{\circ}$, red squares and green triangles- plates with different technologies of superhydrophobic coating, CA $\theta=145^{\circ}$ - show similar effect on the profile

Grant Agency of the Czech Republic, within the project GA101/16-18316S, is gratefully acknowledged for support of this work. Special thanks to Masaryk University, Faculty of Science, Dpt. of Physical Electronics and company ASIO, spol. s r.o.

\section{References}

1. Fialová S., Pochylý F., Identification and Experimental verification of the adhesive coefficient of hydrophobic materials, Wasserwirtschaft Extra, 1/2015, ISSN 0043 0978, pp. 125-129 (2015)
2. Jašíková, D. a Kotek, M.: The Estimation of Dynamic Contact Angle of Ultra-hydrophobic Surfaces Using Inclined Surface and Impinging Droplet Methods. ed. FR: EPJ Web of Conferences, Vol. 67, n. 0. pp. 1 - 6. ISSN 2100-014X (2014)

3. Pochylý F., Fialová, S., Kotek M., Zavadil L., Habán V., Volkov A.V., Parygin A.G., Utilization of hydrophobic layers in the design of hydraulic machines, EkopumpRus'2015 sbornik dokladov, ISBN 978-5-9903138-5-9, printed, pp 77-83 (2015)

4. Jašíková, D., Němcová, L., Kopecký V., The Methodic for Study of Smart Surfaces Using PIV Technique. AIP Conf. Proc. vol. 80, nr. 8. pp. 80 - 87 (2014)

5. Min T. and Kim J.: Effects of hydrophobic surface on stability and transition. Physics of Fluids 17, 108106 (2005)

6. Daniello RJ, Waterhouse NE and Rothstein JP. Drag reduction in turbulent flows over superhydrophobic surfaces. Phys Fluids 21: 085103 (2009)

7. Vinogradova O. I., Slippage of water over hydrophobic surfaces, Int. J. Mineral. Process. 56, pp 31-60 (1999)

8. B.R.K.Gruncelli, N.D.Sandham, G.McHale, Simulations of laminar flow past a superhydrophobic sphere with drag reduction and separation delay, Phys. Fluids 25, 043601, (2013)

9. C. L. M. H. Navier, Mem Acad. Sci. Inst. France, vol. 1, pp. 414-416, (1823).

10. Fialova S., Identification of the properties of hydrophobic layers and its usage in technical practice. Habilitation thesis, VUTIUM. (2016) 